\title{
The necessity of teaching online during the COVID-19 pandemic could result in better Japanese language teaching
}

Edition 7, 2021

Dr Yasuhisa Watanabe

DOI: 10.37839/MAR2652-550X7.15

Moving to online teaching due to COVID-19 related restrictions on face-to-face learning was challenging at first.

Teaching a language subject involves not just vocabulary and grammar, but also how the language is used based on its culture. To be proficient in a language, students need the knowledge of cultural context and manners. Beginners level subjects need to provide instruction on these aspects of language, so students are equipped to study the language to more advanced levels.

The classroom offers the ideal environment for language learning for a number of reasons. Face-to-face contact between students and teachers allows students to engage in conversation practice. Students can receive immediate feedback from their teachers and peers. Teachers can also gauge students' engagement easily from non-verbal cues and adjust their teaching to suit the students' needs. Students' motivation can be kept high by maintaining close peer contact.

The online environment cannot replicate all aspects of classroom language instruction, but online education does have benefits. For example, in asynchronous online teaching, students can learn the materials presented at their own pace without the teaching staff being present. This allows students to spend time on 
content which particularly interests them or content they have difficulty understanding. Allowing students to work in their own space may also allow them to feel freer to take risks, such as speaking aloud.

I argue that in the context of beginners level Japanese language, the quality of learning has improved due to teachers' increased use of online modes of teaching combined with the strengths of face-to-face teaching.

\section{Online language tools can be useful, but don't totally replace face-to-face learning}

It is possible to learn Japanese by watching selected YouTube videos if one is determined enough. An online search using the term 'learn Japanese' on YouTube returns many hits, ranging from a channel run by professional language teachers explaining grammar structures to beginner learners who are recording their learning strategies.

Since the early days of Computer Assisted Language Learning (CALL), the computer has been heralded for its ability to tirelessly ask questions despite having them answered incorrectly. Flashcard or quiz apps to help students memorise vocabulary have been around for over 40 years. More recent apps can selectively ask questions which students answered incorrectly in the past, allowing them to focus on vocabulary they have difficulty memorising without wasting time on items they already know. Teachers can access data on how well students are performing, as well as when, how long, and how often students use the apps, to gauge their level of engagement.

Online quizzes can also help develop listening and reading skills. Questions that require simple answers can be programmed and presented in a similar manner to vocabulary flashcards. Feedback can be tailored to target students' level of understanding. 
Teaching writing in online modes has challenges. Students with a higher level of proficiency-those who already know non-Roman characters and who can construct a meaningful text in the target language-can practice online through email exchanges, bulletin boards and chat tools. However, for students who are learning to put a sentence together, learning to write in the online environment can be a very slow process.

For languages that don't use the Roman alphabet, teaching language-specific scripts is a challenge online due to the reliance on the keyboard to input text. Firstly, the computer must be able to handle Japanese characters with additional software and students must learn how to use the input system. Because there are a far larger number of characters in Japanese than the number of keys on the keyboard, inputting Japanese relies on converting a combined key stroke into one character. Secondly, students must have a fair understanding of how the script system works and how it is translated to the software's conversion system before they can construct a meaningful sentence online. Hand-writing is used more commonly in Japanese classrooms.

In addition to enhancing the effectiveness of learning, hand-writing skills are culturally important in Japanese because one's personality is often judged by their hand-writing skills. Even in the contemporary context where online communication is so prevalent, hand-writing still carries a significant weight in Japanese culture. Important documents, such as an application to open a bank account, are still written by hand. Personal letters are considered more formal when hand-written than typed. When giving a gift, attaching a hand-written note is considered necessary to enhance personal relationships. People who can form hand-written characters in a presentable way are perceived to be more empathetic and caring for others. In order to give students the best possible presentation of themselves, it is considered important to teach the correct formation of hand-written characters in the beginners level Japanese subjects.

When teaching hand writing skills in face-to-face classroom situations, teachers are 
able to correct students' writing whenever they find issues, but this can be very difficult in an online environment. In online teaching, smartphone apps that teach students how to write Japanese characters with their fingers on the screen can be used; and in an online class with a small number of students, it is possible to use students' cameras to show their writing and for teachers to offer individual feedback. However, in a large class this is a very time-consuming practice. Other tools that can help teach writing in an online environment include apps such as Office Lens which can be used to digitise an image of a student's writing so it can be uploaded for teacher feedback. A shared online whiteboard or the draw tool on Padlet can be used synchronously or asynchronously depending on teaching design. But feedback on writing in a classroom setting by a tutor is far superior to what can be offered online. In terms of writing meaningful sentences, online quizzes can accommodate some degree of immediate feedback on students' writing by identifying whether the text is correct or not, but current tools lack the ability to identify where the error is, or give students advice on how to improve.

Some other aspects of face-to-face teaching can also be accommodated online by using synchronous Computer-Mediated Communication (CMC) tools such as Skype and Zoom. However, the level of information conveyed through a computer screen is limited compared to face-to-face communication. Many non-verbal cues such as students' attitudes, demonstrated through body language, are not visible which makes communication more difficult for both students and teachers. Also, many people find the $\mathrm{CMC}$ environment mentally tiring which could be counter-productive.

Perhaps the biggest challenge to teaching languages in the online environment is the lack of social interaction among students. While CMC tools are useful for focused communication, their ability to provide opportunities for social interaction in a large group setting is limited. Language subjects, which require social interaction and discussion as a part of the learning process, can be very difficult to teach well. The lack of social interaction can demotivate students and may be a reason for the low completion rate of online language courses. 


\section{Moving beginners level Japanese subjects online}

My teaching of beginners level Japanese changed a great deal due to COVID-19 restrictions in Australia. I have had to review my face-to-face teaching practices and develop ways to replicate key aspects in the online environment. What has resulted is a 'flipped' classroom, in which students study the teaching contents on their own (self-study) before attending a (shortened) synchronous practice session with a tutor on CMC.

I opted to use short whiteboard animation videos to present parts of the curriculum, which works well in terms of keeping students' attention. I combined the videos with the quiz function of the University of Melbourne's online system for delivering subject content to students (LMS), to replicate a style of teaching that I use in the classroom: asking students questions straight after explaining a new concept to check their understanding. Students were then directed to complete practice exercises in the textbook they would use in face-to-face teaching. The LMS quiz enabled the creation of this sequence of instruction to be completed by students asynchronously. By allowing students to progress at their own pace, some students were able to spend extra time learning without being forced to keep pace with other students. It also reduced the time wasted by high achieving students while they waited for others to catch up.

I taught Japanese scripts via resources available on YouTube made by professional teachers. Students view these instructional video clips, practice their writing on paper and submit it to teachers online for feedback. The instruction is individualised to cater for each student's needs.

Reading and writing comprehension tasks were given to students asynchronously and feedback given at a later stage. Although the ability to provide on-demand feedback while students are working on the task is compromised, the same quality of 
feedback as in a classroom setting can be given if students bring their work to the teacher's attention.

\section{Positive changes in curriculum}

As mentioned, I could not identify much point in keeping the same hours of synchronous teaching online as face-to-face classroom teaching, so I shifted most teaching hours to asynchronous online delivery through video clips and quizzes. After experimenting, I converted each two-hour face-to-face seminar to a combination of one-hour asynchronous online self-study and a one-hour synchronous online seminar. I prepared a structured asynchronous online study resource for each seminar containing several video presentations, revision quizzes, textbook exercises, and listening and reading comprehension tasks. There were also questions to stimulate discussion on aspects of culture, such as how to politely refuse an invitation. The students were tested on their learning during the synchronous online seminar following the asynchronous self-study, and could practice interacting with each other in Japanese, using the knowledge they gained through asynchronous selfstudy. I created a total of 24 sets of self-studies for one semester of teaching.

Assessment was re-designed to support students to become more self-directed learners, instead of relying on a summative test to measure their learning outcomes. Students were asked to keep a portfolio of their learning, following examples used in distance education, that record their goals, homework and reflection on their study each week. Portfolio assessment shifted the weight of assessment from the product of learning to the process of learning, which helps students become more aware of their progress. I have also experimented with a peer-teaching task to reinforce students' preparation and stimulate discussion during the synchronous session. This seemed to improve students' overall achievement despite having less contact hours with teachers. 


\section{More diverse interaction}

My online curriculum aimed to promote three types of interaction online: teacherlearner, learner-learner, and learner-content.

In face-to-face classroom teaching before COVID-19, the main type of interaction in the beginners level Japanese subjects was teacher-learner interaction, where the classroom teacher had responsibility for delivering the curriculum. Learner-learner interaction (such as peer practice) and learner-content interaction (such as preparing for each class using textbooks) were left to students' discretion. Many students were reliant on the teacher presenting the instruction to them rather than proactively learning the contents before the class.

Moving to online teaching and introducing asynchronous self-study encouraged students to engage in learner-content interaction. Because students were engaging in preparation before synchronous online seminars, learner-learner interaction during synchronous online sessions became more active and student motivation remained high.

Scores from asynchronous self-study were useful for teachers to gauge students' engagement levels. The information was also used to compose relevant questions for students at the start of synchronous online seminars, which promoted teacherlearner interaction.

\section{Types of engagement promoted}

In pre-COVID face-to-face classroom teaching, cognitive engagement, which refers to students thinking about the materials at a deeper level, was promoted by the teacher's presence. But I found in online teaching that this could be achieved through asynchronous online content with which students could engage at their own pace. Pairing video presentations with online quizzes ensures students reflect on what is presented immediately after viewing. Portfolio assessment, which asks 
students to set their own goals, compile their work and reflect on their achievements also promotes cognitive engagement.

\section{Student-centred learning design}

I experimented with a group assignment task using inquiry-based learning to promote student-centred learning. Designed as a group presentation, students were asked to teach a grammar lesson to the rest of the class in place of the tutor. They were required to research the designated grammar structure, explain it to the class with sample dialogues to demonstrate how they are used in conversation, and lead a practice session with their peers. While some groups only managed to present the basic contents, others were creative and highly effective. Some students were adept at using online shared documents to organise collaborative work and devised a related online competitive game to bring in an aspect of fun into their teaching. In some instances, the presenters were challenged by the audience on the contents of their teaching, stimulating further discussion and experiments with the new grammar structure. Using peer presentations to introduce learning content may be common in other areas such as science and business, but it was a new experiment to try this in a language subject. Students had to upskill themselves before they could make a presentation, which made them engage with the contents more deeply. This assessment is something I would like to keep in face-to-face teaching.

\section{A new curriculum design}

What I have done with the beginners level Japanese subjects during COVID-19 restrictions on face-to-face learning is to rearrange the existing curriculum using online technology. But face-to-face teaching should retain the effective aspects of online teaching. The best way forward for beginners level Japanese subjects would be a new curriculum design that uses the strengths of both modes of teaching.

Teaching in both synchronous and asynchronous modes has advantages. The 
asynchronous presentation of contents provides students with more flexibility. Teachers can easily monitor students' progress in the online environment and faceto-face teaching time can then be used to follow up on the areas in which students are having difficulties. Learning can be streamlined for both students and the teaching team. Many students are more engaged and develop a greater sense of responsibility for their learning, when assessment is focused on the process of learning rather than just outcomes.

Aspects of online teaching could be adapted to create new subjects, such as fully online courses for students who are not able to commute to campus. With limited face-to-face components and shorter intervals between seminars, current subjects could also be transformed into an intensive subject. Both could be implemented without much more investment to attract more students.

COVID-related lockdowns have forced us rethink and be creative about how language subjects can be taught well online. What we've learned can make language teaching greater.

Image credit: Dominic Alves/Flickr. This image has been cropped. 